\title{
Questes
}

vestes Revue pluridisciplinaire d'études médiévales

\section{Naissances : introduction}

\section{Émilie Deschellette et Céline Ménager}

\section{OpenEdition}

Journals

Édition électronique

URL : http://journals.openedition.org/questes/751

DOI : 10.4000/questes.751

ISSN : 2109-9472

\section{Éditeur}

Les Amis de Questes

\section{Édition imprimée}

Date de publication : 15 janvier 2014

Pagination : 13-34

ISSN : 2102-7188

\section{Référence électronique}

Émilie Deschellette et Céline Ménager, « Naissances : introduction», Questes [En ligne], 27 | 2014, mis en ligne le 15 janvier 2014, consulté le 25 septembre 2020. URL : http://journals.openedition.org/ questes/751 ; DOI : https://doi.org/10.4000/questes.751 


\title{
Introduction
}

\section{Émilie DesChellette et Céline MÉNAGer}

\author{
Université Paris-Sorbonne (Paris IV)
}

«Jésus répondit $[\ldots]$ : "En vérité, en vérité, je te le dis : si quelqu'un ne naît de nouveau, il ne peut voir le royaume des cieux". Nicodème lui dit: "Comment un homme peut-il naître lorsqu'il est vieux ? Est-ce qu'il peut entrer de nouveau dans le sein de sa mère et renaître ?" "1"

«On dit que la naissance vous fait oublier les temps antérieurs ; on dit que mettre au jour, c'est mettre à jour, comme un carnet qu'on fait débuter à blanc en lui arrachant des pages $^{2} \gg$.

Venir au monde : c'est, a priori, l'acte le plus universel et « naturel» qui soit. Venir à l'existence : c'est, a priori, le lieu du passage du néant à l'être. Avec la naissance, ce qui n'était pas encore prend forme, corps et sens. Or, 1'action de naître se présente d'emblée tissée de paradoxes : 1'on ne saurait la réduire au moment ponctuel et bien circonscrit de l'accouchement, car toute naissance est en réalité « en germe », elle s'annonce et se prépare, faisant l'objet d'une conception, au sens biologique du terme, mais également d'une construction, dans le domaine des représentations. Il nous faut donc associer, pour véritablement la comprendre, à la venue au monde proprement dite, le processus de conception dont elle dépend en amont, ainsi que l'inscription dans la

\footnotetext{
${ }^{1}$ Vulgate, Évangile de Jean, 3, 3-4 : « Respondit Jesus [...] : Amen, amen dico tibi, nisi quis renatus fuerit denuo, non potest videre regnum Dei. Dicit ad eum Nicodemus : Quomodo potest homo nasci, cum sit senex ? numquid potest in ventrem matris suæ iterato introire et renasci ? ", La Sainte Bible (texte latin et traduction française), commentée, d'après la Vulgate et les textes originaux, à l'usage des séminaires et du clergé par Louis-Claude Fillion, t. 7 «Les Saints Évangiles. Les Actes des apôtres », Paris, Letouzey, 1921.

${ }^{2}$ André Pieyre de Mandiargues, Porte dévergondée, Paris, Gallimard, 1965, p. 149.
} 
société qu'elle engage en aval. Si, par son caractère charnel, la naissance rappelle à l'Homme sa corporéité et son appartenance à l'ordre naturel du vivant, elle transcende largement cette seule dimension biologique ${ }^{3}$. Ce que Maurice Godelier affirmait à propos de la sexualité nous paraît également éclairer le fonctionnement de la naissance : «machine ventriloque à travers laquelle la société parle sur elle-même ${ }^{4}$ », la naissance est un fait éminemment social; les représentations qu'elle génère sont donc axiologiquement marquées. Les théories sur la conception, le partage des fonctions et la responsabilité incombant respectivement à l'homme et à la femme dans la venue au monde d'un enfant, varient en effet d'une société à l'autre, dans l'espace et dans le temps ${ }^{5}$. Le rôle qu'une société donnée attribue à chacun des parents, dans le processus qui conduit de l'engendrement à l'émergence de la vie, révèle et détermine l'organisation $\mathrm{du}$ groupe social. Les conceptions de la naissance entrent ainsi en résonnance avec les représentations du corps, de la parenté et de la personne humaine, formant un réseau complexe. Pour reprendre les termes d'Anita Guerreau-Jalabert, dans son très éclairant article sur le baptême, «les représentations de l'homme portent témoignage des structures sociales, qui se trouvent ainsi en quelque sorte inscrites en tout individu et fondées en nature ${ }^{6} »$. Il existe en effet des corrélations entre les conceptions de l'engendrement charnel et les systèmes de parenté, bien que ces

\footnotetext{
${ }^{3}$ Voir à ce sujet l'ouvrage de Didier Lett, Hommes et femmes au Moyen Âge. Histoire $d u$ genre, $X I I^{e}-X V^{e}$ siècle, Paris, Armand Colin, coll. "Cursus Histoire », 2013, en particulier le chapitre 2, « Les corps sexués », p. 27-40.

4 Maurice Godelier, «L'Occident, miroir brisé. Une évaluation partielle de l'anthropologie sociale assortie de quelques perspectives ", Annales Économies, Sociétés, Civilisations, $48^{\mathrm{e}}$ année, $\mathrm{n}^{\mathrm{o}}$ 5, 1993, p. 1183-1207, p. 1191.

${ }^{5}$ Maurice Godelier présente des analyses extrêmement éclairantes à ce sujet dans son article « L'Occident, miroir brisé...», art. cit., p. 1190 et suiv.

${ }^{6}$ Anita Guerreau-Jalabert, «Spiritus et caritas. Le baptême dans la société médiévale », dans La Parenté spirituelle, éd. Françoise Héritier-Augé, Elisabeth Copet-Rougier, Paris, Édition des Archives contemporaines, 1996, p. 133-203.
} 
« correspondance[s] [ne soient] jamais mécanique[s] ${ }^{7}$ ». De fait, si l'on adopte la définition que Maurice Godelier propose de la parenté - qu'il nous faudra cependant préciser pour rendre compte des spécificités des conceptions médiévales à cet égard -, l'on perçoit bien que la naissance est au cœur de ces enjeux : pour l'anthropologue, en effet, la parenté recouvre «l'ensemble des principes qui définissent des unions légitimes entre individus des deux sexes et déterminent identité et appartenance des enfants qui naissent de ces unions ${ }^{8} »$. La naissance porte ainsi l'empreinte des relations de parenté, et de façon plus large, des « rapports sociaux, politiques et religieux ${ }^{9} »$. La naissance s'inscrit dans un réseau de représentations, qui structurent la société et l'univers, s'accompagne de rites et de rituels, et s'incarne dans des discours complexes ${ }^{10}$. La naissance est en effet au croisement de la réalité concrète et physiologique de l'engendrement, des conceptions relatives à la reproduction de l'espèce et de la parenté, conçue comme « fait social ${ }^{11} »$.

\footnotetext{
${ }^{7}$ Maurice Godelier, « L’Occident, miroir brisé...», art. cit, p. 1191.

${ }^{8}$ Maurice Godelier, «L'Occident, miroir brisé...», art. cit., p. 1191-1192. Il avance cependant la nuance suivante : «Cette définition n'est pas nécessairement acceptée par tous les anthropologues et David Schneider dans A Critique of the Study of Kinship (1984) rejette totalement idée que la parenté soit universellement liée aux processus de conception et appropriation des enfants ", ibid.

${ }^{9}$ Maurice Godelier, «L'Occident, miroir brisé...», art. cit., p. 1191

${ }^{10}$ Voir notamment L'Imaginaire de la parenté dans les romans arthuriens (XII $-X I V^{e}$ siècles), éd. Martin Aurell et Catalina Girbea, coll. « Histoires de famille. La parenté au Moyen Âge », 11, Actes du colloque international du Centre d'Études Supérieures de Civilisation Médiévale de l'Université de Poitiers (12 et 13 juin 2009), Turnhout, Brepols, 2010.

${ }^{11}$ Jérôme Baschet, « La Parenté partagée : engendrement charnel et infusion de l'âme (à propos d'une miniature de la fin $\mathrm{du} \mathrm{XV}^{\mathrm{e}}$ siècle) », p. 123, note 2 . Il précise que, «comme l'enseigne l'anthropologie, les systèmes de parenté sont toujours une élaboration sociale des données que nous dirions biologiques", et convoque également à ce sujet les réflexions de Françoise Héritier : "Toute parenté est sociale, car elle consiste essentiellement en relations juridiques et morales sanctionnées par la société »" (Françoise Héritier, L'Exercice de la parenté, Paris, Gallimard/Le Seuil, coll. «Hautes études », 1981, p. 14-15. Dans la même perspective, voir son ouvrage Masculin/Féminin. La pensée de la différence, Paris, Éditions Odile Jacob, 1996.
} 
Dans ce cadre, le détour par l'étymologie n'est pas dépourvu d'intérêt : le substantif naissance, attesté à partir du XII ${ }^{\mathrm{e}}$ siècle, est en effet un dérivé du verbe nasci, «naître» au sens propre et "prendre son origine » au sens figuré, issu de ${ }^{\circ}$ gnasci, qui repose sur une base ${ }^{\circ} g n a-$ apparentée, bien que cette origine ne soit plus sentie en latin même, à la racine indo-européenne ${ }^{\circ}$ gen-, "engendrer" et "naître"12 ". Or cette base, qui associe la venue au monde, au sens strict, et le processus de conception qui intervient en amont, «exprime surtout la descendance, $[\ldots]$ la parenté reconnue, puis un groupe social fondé sur la parenté ». Le mot naissance est par ailleurs très tôt employé avec les sens figurés de "moment où apparaît, où commence quelque chose » et « début, origine », déjà contenus dans le sème du verbe latin. Ce dense réseau de significations nous permet ainsi d'envisager le terme dans ses diverses acceptions et de lui adjoindre les notions de «génération » et de «création », de façon à embrasser l'ensemble de la palette sémantique que recèle l'idée de «naissance ».

Quelques précautions d'ordre conceptuel s'imposent néanmoins. Dans un article consacré à la création d'Ève, Jérôme Baschet attire l'attention sur la nécessité de «questionner notre propre regard contemporain, prodigue en métaphores de l'engendrement et de la naissance ${ }^{13} »$ et de se garder de les appliquer sans distance critique aux conceptions du Moyen Âge. Il explique que si la parenté, à la fois « charnelle, spirituelle et divine » est très présente à l'époque médiévale, elle n'y a le statut «ni de métaphores, ni de fantasmes, mais d'éléments bien réglés d'un ensemble de représentations ${ }^{14} »$.

12 Dictionnaire historique de la langue française [1992], dir. Alain Rey, Paris, Dictionnaires Le Robert, 2004, t. 2, p. 2338, article « Naître ».

${ }^{13}$ Jérôme Baschet, « Ève n'est jamais née. Les représentations médiévales et l'origine du genre humain ", Ève et Pandora. La création de la première femme, dir. Jean-Claude Schmitt, Paris, Gallimard, 2002, p. 115-162, p. 161.

14 Jérôme Baschet, « Ėve n'est jamais née... », art. cit., p. 162. 
Le Moyen Âge illustre en effet d'une manière tout à fait spécifique ce que Maurice Godelier rappelle au sujet de la génération et de ses représentations : «nulle part [...] un homme et une femme ne suffisent [à eux seuls] à faire un enfant ${ }^{15} »$. Il va nous falloir approfondir cet aspect, dont nous nous contentons pour lors de poser les jalons. Les fondements théologiques sur lesquels repose le christianisme densifient et complexifient les conceptions de l'engendrement - dont relève la naissance -, en faisant se rencontrer sphère charnelle et sphère spirituelle. À la question de la reproduction du corps et de l'intervention différenciée de l'homme et de la femme s'ajoute en effet celle de la formation de l'âme et de son « infusion », par un troisième actant, Dieu, lors de la naissance ou en amont de celle-ci. Par ailleurs, pour le christianisme, la naissance biologique, sortie de la matrice maternelle, ne suffit pas: la seconde naissance, ou « renaissance », que constitue le baptême est indispensable pour véritablement venir au monde et exister aux yeux de la société. Par le baptême, l'on renaît, non plus seulement comme membre de l'espèce humaine et comme l'enfant de ses parents charnels, mais comme membre de la communauté chrétienne et enfant de Dieu. Enfin, la génération charnelle tend à être transcendée par des engendrements spirituels, en

${ }^{15}$ Maurice Godelier, Au fondement des sociétés humaines. Ce que nous apprend l'anthropologie, Paris, Flammarion, coll. «Essais », 2010, p. 158. L'anthropologue explique que «quel que soit le système de parenté, patrilinéaire, matrilinéaire, indifférencié etc., quelle que soit la religion dominante, polythéiste ou monothéiste, etc., les sociétés avaient développé des théories de la fabrication des enfants qui toutes supposaient qu'un homme et une femme par leurs rapports sexuels (et par les substances qu'ils peuvent apporter, ajouter ou mélanger), font seulement des fœtus qui ne sont pas encore des enfants. [...] Partout on suppose que des agents autres que les humains et plus puissants qu'eux, interviennent [...]. Par exemple le fotus recevra son souffle d'un Dieu et son âme d'un ancêtre qui se réincarne en lui. Si vous faites le compte, pour faire un enfant il faut alors un homme, une femme, un ancêtre et un Dieu ». Entretien de Maurice Godelier avec Maryvonne Barraband, Xavier Gassmann et Françoise Petitot, «Un homme et une femme ne suffisent pas à faire un enfant », La Lettre de l'enfance et de l'adolescence, $\mathrm{n}^{\circ}$ 59, 2005, p. 17-26, p. 17. Source : http://www.cairn.info/revue-lettre-de-l-enfance-et-de-1-adolescence-2005-1page-17.htm (page consultée le 30 janvier 2014). 
particulier au regard du mystère de l'Incarnation, en relation avec le dogme trinitaire. Le Concile de Nicée de 325, qui condamne Arius et sa doctrine, entérine en effet que le Fils est « engendré, non pas créé », de même nature que le Père et consubstantiel à Dieu. Quant à la théologie mariale, elle proclame la virginité absolue d'une mère, avant, pendant et après la naissance de Christ. L'interprétation de la Bible, des mystères et des sacrements sont des «données importantes dans les conceptions de la reproduction sociale et dans l'organisation de l'Église $»^{16}$. Les conceptions médiévales de la naissance revêtent en effet des formes institutionnelles et s'inscrivent dans un système idéologique et symbolique dont il faut mesurer le fonctionnement spécifique.

Les enjeux engagés par ces questions expliquent que les discours théologiques médiévaux se caractérisent par un usage relativement rigoureux du vocabulaire de la génération, et il nous faut tenter de situer le processus de la naissance au regard des autres termes qui s'offrent pour dire l'accès à l'existence, et avec lesquels il entre en résonnance sémantique et conceptuelle. Les scolastiques distinguent en effet quatre opérations qui font advenir les êtres ou les choses à l'existence : «faire, comme l'artisan qui fabrique des objets à partir d'une matière extérieure à lui ; créer, qui consiste à faire à partir de rien, engendrer, c'est-à-dire faire soi-même de sa propre substance, en instituant un rapport de filiation; procéder, [...] suppose une également une idée de substance, sans être pour autant un engendrement ${ }^{17} \gg$. Les développements que Thomas d'Aquin consacre dans sa Somme théologique à ce sujet sont révélateurs de l'enjeu que constitue cette épineuse question :

Distinguons deux emplois du mot «génération ». On l'applique d'abord dans un sens général à tout ce qui s'engendre et se corrompt; dans ce cas,

\footnotetext{
${ }^{16}$ Anita Guerreau-Jalabert, «Spiritus et caritas... », art. cit., p. 160.

${ }^{17}$ Jérôme Baschet, « Ève n’est jamais née... », art. cit., p. 141.
} 
« génération » ne signifie rien d'autre que le passage du non-être à l'être. Nous en usons en second lieu, et cette fois au sens propre, à propos des vivants ; dans ce cas, "génération » signifie « l'origine qu'un vivant tire de son principe vivant conjoint $»$ : on la nomme proprement « naissance ». Cela pourtant ne suffit pas pour être qualifié d' «engendré »; ce nom n'est donné proprement qu'à ce qui procède selon la ressemblance au principe ${ }^{18}$.

Par ailleurs,

entre « engendrer » vraiment, acte par lequel un fils procède, et « faire », il y a cette différence, que l'on fait une chose avec une matière extérieure ; le menuisier fait un escabeau avec du bois, mais c'est de sa propre substance que l'homme engendre un fils. Et tandis que l'artiste créé fait quelque chose d'une matière donnée, Dieu, lui, fait quelque chose de rien ${ }^{19}$.

Les contributions de ce bulletin illustrent bien les paradoxes que recèle la naissance et la densité du réseau sémantique dans lequel elle s'inscrit, engageant des enjeux tout à la fois idéologiques, sociaux et poétiques. Il s'agit d'abord d'envisager la naissance comme lieu de passage et de fondation, pour ensuite interroger la temporalité complexe de la

18 Thomas d'Aquin, Summa Theologiae, Prima Pars, quaestio 27, articulum 2: « sciendum est quod nomine generationis dupliciter utimur. Uno modo, communiter ad omnia generabilia et corruptibilia, et sic generatio nihil aliud est quam mutatio de non esse ad esse. Alio modo, proprie in viventibus, et sic generatio significat originem alicuius viventis a principio vivente coniuncto. Et haec proprie dicitur nativitas. Non tamen omne huiusmodi dicitur genitum, sed proprie quod procedit secundum rationem similitudinis », Summa theologiae cum Supplemento et commentariis Caietani, Rome Textum Leoninum Romae, 1886-1906, mis en ligne par le Corpus Thomisticum de la Fundación Tomás de Aquino: http://www.corpusthomisticum.org/index.html (page consultée le 25 janvier 2014). Thomas d'Aquin, Somme théologique, traduction Aimon-Marie Roguet, coordination Albert Raulin, Paris, Éditions du Cerf, 4 vol., 1984-1986, vol. 1.

${ }^{19}$ Ibid., Prima Pars, quaestio 41, articulum 3 : « Hoc autem interest inter generationem veram, per quam aliquis procedit ut filius, et factionem, quod faciens facit aliquid de exteriori materia, sicut scamnum facit artifex de ligno; homo autem generat filium de seipso. Sicut autem artifex creatus facit aliquid ex materia, ita Deus facit ex nihilo », éd. et trad. cit. 
naissance, faite de déplacements et de dilatations. Il nous faudra enfin questionner les modèles, parfois ambigus et protéiformes, que convoque le Moyen Âge pour représenter et écrire la naissance.

\section{La naissance, moment de passage et de transition}

La naissance est le lieu où se cristallise toutes les tensions : les représentations de la naissance déplacent en effet les frontières, entre sphère privée et cadre de la polis, entre nature et culture, entre ce qui relève du masculin et du féminin, entre charnel et spirituel, enfin.

La naissance est un moment de passage pour l'enfant, qui vient au monde, comme pour l'homme et la femme, qui acquièrent, avec plus ou moins d'évidence, le statut de père et de mère ${ }^{20}$. Ce passage s'accompagne de rites et de discours, nécessaires pour intégrer le nouvel être dans la société : il s'agit en effet de donner du sens à l'évènement de la naissance, par des gestes et des mots qui inscrivent dans la culture ce qui relève également de la nature, prouvant, si besoin était, le caractère équivoque de cette dichotomie. La naissance fait bien souvent l'objet d'un retour réflexif ou d'une (re)construction a posteriori. C'est pourquoi la naissance peut être exploitée à des fins politiques ou idéologiques, notamment dans une perspective généalogique.

La naissance, de fait, ne va pas de soi, et cela est d'autant plus vrai au Moyen Âge, où la promesse de vie qu'elle constitue a priori est pétrie d'incertitudes, l'issue de l'accouchement s'avérant bien souvent funeste, pour le nouveau-né comme pour la mère. Des médiateurs accompagnent donc les deux protagonistes dans le passage périlleux qu'est la naissance : l'intervention de la sage-femme, et plus largement de la communauté féminine, qui assistent la future mère et prennent soin de l'enfant, est

\footnotetext{
${ }^{20}$ On connaît bien la maxime « Mater semper certa est, pater semper incertus est ».
} 
indispensable ${ }^{21}$. Dans ce bulletin, Céline Ménager lève le voile de la courtine pour examiner les realia de la naissance en pénétrant dans « la chambre de l'accouchée $»^{22}$. Néanmoins, l'assistance médicale purement humaine ne suffit pas et l'on sollicite également des saints - plus souvent des saintes, d'ailleurs - pour qu'ils intercèdent au moment de la naissance. C'est le cas de Marguerite d'Antioche, présentée comme la protectrice des parturientes, invitées à toucher sa ceinture pour s'assurer un accouchement heureux ${ }^{23}$.

La naissance pourrait ainsi sembler relever avant tout de la sphère féminine, mais c'est sans compter le partage spécifique des rôles qu'institue le Moyen Âge: «Dans la société médiévale, ce sont les hommes qui engendrent. Au-delà des divergences entre les héritages galénique et aristotélicien, il apparaît clairement que les hommes jouent le rôle principal dans la reproduction humaine, comme l'indique le partage lexical tendanciel qui leur attribue le pouvoir d'engendrer (gignere) tandis que le rôle des femmes se limite à concevoir (concipere, terme qui suggère le rôle passif de réceptacle) et à enfanter (parere) ${ }^{24} »$, terme qui renvoie à l'accouchement.

Pour l'homme comme pour la femme, cela dit, la naissance est perçue comme un moment de fondation, que ce soit la fondation d'une

${ }^{21}$ Elle est d'ailleurs bien représentée dans l'iconographie : à titre d'exemple, voir les miniatures illustrant ce bulletin.

${ }^{22}$ Voir Céline Ménager, «Dans la chambre de l'accouchée : quelques éclairages sur le déroulement d'une naissance au Moyen Âge », infra, p. 35-45.

${ }^{23}$ De nombreuses fontaines consacrées à sainte Marguerite étaient le lieu de rituels liés à l'enfantement: "les femmes venaient suspendre à leurs abords des vêtements d'enfant, pour placer leur fertilité [...] et leurs accouchements sous [sa] protection » (Martine Creusy-Chopard, "L'image de sainte Marguerite d'Antioche: un imaginaire remis en question », article en ligne sur Imageson.org, avril 2005, http://www.imageson.org/document511.html, page consultée le 31 janvier 2014). Sur la légende de sainte Marguerite, voir notamment Raban Maur, Martyrologium, éd. John McCulloh, Corpus Christianorum Continuatio Mediaeualis, XLIV, Turnhout, Typographi Editores Pontificii, 1979, p. 67-68.

${ }^{24}$ Jérôme Baschet, « Ève n'est jamais née... », art. cit., p. 138. 
famille, ou plus spécifiquement, la fondation d'une dynastie, des liens sociaux s'y nouent et des enjeux de légitimité s'y jouent. Il ne s'agit pas seulement de concevoir un enfant, mais également de reconnaître un nouveau membre de la communauté, de l'inscrire dans son réseau de parenté et, par là, dans la société. Au cours des $\mathrm{XI}^{\mathrm{e}}-\mathrm{XIII}{ }^{\mathrm{e}}$ siècles, l’Église veille précisément à encadrer plus rigoureusement l'alliance matrimoniale des laïcs, pour endiguer la sexualité, et par suite, la procréation. Le souci de la société médiévale de contrôler la naissance s'explique par la volonté de maîtriser la filiation, menacée par le soupçon qui pèse sur l'engendrement, et qui peut par conséquent entacher la généalogie.

L'on comprend donc que la naissance soit génératrice de tabous et d'interdits. Les problématiques que soulèvent les naissances qui surviennent hors des cadres du mariage établis par l'Église sont multiples, de la question des enfants « naturels » à celle de la bâtardise. Elles mettent en effet au jour des rapports de force complexes, dont la perception et les implications sont loin d'être univoques. Les frontières juridiques et sociales en matière de filiation illégitime et de bâtardise, sont particulièrement délicates à définir, parce que mouvantes d'un point de vue spatio-temporel, alors même qu'elles déterminent la régulation du groupe social ${ }^{25}$. Nous nous permettons de renvoyer à ce sujet aux recherches que conduit actuellement le CERHIO d'Angers ${ }^{26}$. La naissance, de même que le

25 Voir, entre autres, Anne Lefebvre-Teillard, «L'enfant naturel dans l'ancien droit français ", Recueil de la société Jean Bodin, 36/2, 1976, p. 253-264 ; Michaël Harsgor, "L'essor des bâtards nobles au XV siècle », Revue Historique, 253, 1975, p. 319-354; Bastardy and its Comparative History. Studies in the history of illegitimacy and marital nonconformism in Britain, France, Germany, Sweden, North America, Jamaica and Japan, dir. Peter Laslett, Karla Oosterveen and Richard M. Smith, London, Arnold, 1980. Nous renvoyons également à notre bibliographie en fin de bulletin.

${ }^{26}$ Il s'agit du projet «Filiation illégitime et bâtardise dans les sociétés médiévale et moderne : histoire, droit, lettres », dont on peut consulter la présentation par Carole Avignon à l'adresse suivante : http://confluences.hypotheses.org/307 (page consultée le 30 janvier 2014). Voir également notre bibliographie en fin de bulletin. 
baptême, (re)naissance «ex aqua, et Spiritu Sancto $^{27}$ ", sont de ces «moments de la vie sociale durant lesquels [les] questions d'identification ou de fama [se] pos[ent] : abandons ou infanticides, modalités d'intégration dans un groupe domestique [...] et [d']insertion dans l'ecclesia ${ }^{28} »$.

L'instabilité des discours sur la bâtardise est par ailleurs manifeste dans la fiction, où elle constitue un ressort diégétique extrêmement fécond : la naissance illégitime fait l'objet de variations axiologiques qui ont été finement analysées, notamment pour les figures ambigües de Charlemagne, d'Alexandre ou d'Arthur ${ }^{29}$. Pour peu que la bâtardise soit le fruit d'une Providence, qui transcende la seule volonté des parents et l'acte charnel humain, elle peut devenir vertu et se faire le signe d'un destin exceptionnel, appelant bien souvent l'enfant né de cet accouplement illégitime à fonder, paradoxalement, l'identité d'un groupe.

En contre-point de ces unions à la marge, mais fécondes, comment vivre la stérilité, à une époque de pouvoir dynastique ? Comment, dans une société où la femme n'est véritablement qu'en devenant mère, acquérir un statut, exister aux yeux de la communauté, lorsque la naissance se dérobe et se refuse? C'est ce qu'interroge Stéphanie Richard dans ce bulletin, en analysant le procès en nullité du mariage de Louis XII et Jeanne de France $^{30}:$ le couple incapable de procréer se voit menacé dans sa durée.

L'union a priori indissoluble qui attache les époux l'un à l'autre est en effet sous-tendue par des fondements théologiques qui puisent leurs

${ }^{27}$ Vulgate, éd. cit., Évangile de Jean, 3, 5.

${ }^{28}$ Carole Avignon, page citée.

${ }^{29}$ Voir les articles de Dominique Boutet, « Bâtardise et sexualité dans l'image littéraire de la royauté $\left(\mathrm{XII}^{\mathrm{e}}-\mathrm{XIII}{ }^{\mathrm{e}}\right.$ siècles) », Femmes, Mariages-Lignages, XII ${ }^{e}-\mathrm{XIV}^{e}$ siècles, Mélanges offerts à Georges Duby, Bruxelles, De Boeck, 1992 et de Laurence Mathey-Maille, «Le roi Arthur chez Geoffroy de Monmouth et Wace : la naissance du héros ", Arturus Rex. Acta Conventus Lovaniensis 1987, éd. Willy van Hoecke, Gibert Tournoy, Werner Verbeke, Leuven, Leuven University Press, Series I/Studia XVII, 1991, p. 222-228.

${ }^{30}$ Voir Stéphanie Richard, «Sans naissance, pas de mariage ? Le procès en nullité du mariage de Louis XII et Jeanne de France (1498) », infra, p. 47-66. 
sources dans l'Écriture. La procréation qui légitime le mariage, et la naissance qui en est l'aboutissement attendu, se trouvent ainsi déplacées dans la sphère du sacré, au sein d'une corrélation complexe entre dimensions charnelle et spirituelle ${ }^{31}$. La sexualité et l'engendrement charnels, entachés par le péché originel, sont transcendés par la nouvelle naissance que constitue le baptême et qui rachète la souillure des premiers parents de l'humanité, transmise par la génération. Bien entendu,il ne s'agit pas de proposer ici une analyse exhaustive de cet aspect fondamental du dogme chrétien ${ }^{32}$ : ce que l'on en retient pour notre objet, c'est ce qui fait de la naissance le lieu d'un passage ; passage de la chair de la conception sexuelle à l'esprit que Dieu insuffle dans le baptisé, passage du sang du ventre maternel à l'eau qui purifie, passage de la famille privée à une communauté, où tous les chrétiens sont fils de Dieu et de l'Église ${ }^{33}$. «Le baptême matérialise [ainsi] la domination de l’Église dans un rite de reproduction sociale qui associe, avec un décalage hiérarchique, prêtre et

${ }^{31}$ Jean-René Valette examine le caractère opérant de ces catégories conceptuelles dans le roman arthurien dans son article «Filiation charnelle et adoption filiale: l'imaginaire de la paternité dans la Queste del Saint Graal», L'Imaginaire de la parenté, op. cit., éd. Martin Aurell et Catalina Girbea, p. 47-59.

${ }^{32}$ Nous renvoyons pour cela à Anita Guerreau-Jalabert, art. cit.

${ }^{33}$ Dans l'Évangile de Jean, les questions de Nicodème à Jésus soulèvent précisément le paradoxe d'une «renaissance»: «Quomodo potest homo nasci, cum sit senex ? numquid potest in ventrem matris suæ iterato introire et renasci ? Vulgate, éd. cit., Évangile de Jean, 3, 4 (« Comment un homme peut-il naître lorsqu'il est vieux ? Estce qu'il peut entrer de nouveau dans le sein de sa mère et renaître ? », trad. cit). Or, Jésus, en affirmant la suprématie de la renaissance spirituelle sur la naissance charnelle, indique bien qu'il s'agit de dépasser les cadres de la génération humaine ordinaire : " nisi quis renatus fuerit ex aqua, et Spiritu Sancto, non potest introire in regnum Dei. Quod natum est ex carne, caro est : et quod natum est ex spiritu, spiritus est. Non mireris quia dixi tibi : oportet vos nasci denuo », op. cit., Évangile de Jean, 3, 5-7 (« si quelqu'un ne renaît de l'eau et de l'Esprit saint, il ne peut entrer dans le royaume de Dieu. Ce qui est né de la chair est chair, et ce qui est né de l'esprit est esprit. Ne t'étonne pas de ce que je t'ai dit : il faut que vous naissiez de nouveau ", trad. cit.). 
laïcs dans l'affirmation du spirituel sur le charnel comme principe structurant de l'homme et de l'organisation sociale ${ }^{34} »$.

Génération et naissance reposent ainsi au Moyen Âge sur une dichotomie charnel/spirituel qui vient recouper la bipartition genrée, hiérarchisant de façon complexe les rôles de l'homme et de la femme. Ce sont ces tensions qu'analyse Thibault Radomme à travers les « enfantements charnel et spirituel ${ }^{35}$ » dans la correspondance d'Abélard et Héloïse. Comme le rappelle Jérôme Baschet,

Engendrer est un privilège que les figures masculines n'accaparent pas seulement dans le domaine charnel, puisque les prêtres sont considérés comme les agents visibles de l'engendrement spirituel des chrétiens dans le baptême. Enfin, on ne saurait oublier que le Père divin est le géniteur suprême, non seulement parce que tous les chrétiens sont ses enfants spirituels, mais aussi parce qu'il est le seul être capable de produire l'engendrement parfait de son Fils ${ }^{36}$.

La domination masculine dans le domaine de la naissance tout à la fois biologique, spirituelle et sociale, se retrouve également lorsque l'on envisage la création poétique. Barbara Falleiros soulève en effet la question, dans son article consacré à la construction d'une figure de femme écrivain par Christine de Pizan ${ }^{37}$. La quête de ce statut nous paraît passer, d'une façon tout à fait intéressante, successivement par une naissance, puis une renaissance : cette ambigüité et ce glissement pourraient faire écho, métaphoriquement, aux conceptions que nous avons évoquées. C'est d'abord invoquant un modèle masculin - celui de son père - que Christine

\footnotetext{
${ }^{34}$ Anita Guerreau-Jalabert, «Spiritus et caritas... », art. cit., p. 171.

${ }^{35}$ Voir la contribution de Thibaut Radomme dans ce bulletin, « De "la maleiçon d'Eve en la beneiçon Marie": enfantements charnel et spirituel dans la correspondance d'Abélard et Héloïse », infra, p. 67-87.

${ }^{36}$ Jérôme Baschet, « Ève n'est jamais née », art. cit, p. 138.

${ }^{37}$ Voir dans ce bulletin, Barbara Falleiros, «Génération et création poétique : la naissance d'une femme écrivain », infra, p. 89-108.
} 
de Pizan revendique une légitimité créatrice, mais ce modèle n'est en définitive qu'une transition vers l'affirmation de son statut de femme créatrice. Dans son analyse de l'incipit de Cléomadès, Aurélie Houdebert montre par ailleurs qu'Adenet de Roi met en avant, dans la conception et la naissance de son œuvre, le rôle de deux femmes, Marie de Brabant, épouse de Philippe III, et sa belle-sœur, Blanche de France : la mise en scène du processus créatif à l'orée du roman oscille ainsi subtilement entre éloge des commanditaires féminins et valorisation de l'écrivain au travail, chargé de mettre l'œuvre au monde ${ }^{38}$.

Si la naissance, enfin, se présente comme un passage, que l'on cherche à circonscrire dans des cadres, juridiques comme idéologiques, c'est qu'elle demeure un phénomène qui échappe en partie à l'homme. D’un point de vue épistémologique, en effet, «tout commencement, [...] relatif comme [...] absolu, demeure insaisissable comme tel ». «Événement pour la mère, le personnel d'accouchement, les témoins, [la naissance] ne l'est pas pour celui qui nait ${ }^{39} »$ : elle échappe à la fois à l'expérience et à la conscience. Notre propre naissance n'est jamais en définitive vécue que par autrui.

C'est sans doute ce caractère insaisissable qui permet, au moment de la venue au monde, le jaillissement de la faute, du merveilleux ou de l'étrange, ce qu'illustrent notamment les signes que portent certains enfants dont la conception relève de l'extra-ordinaire et la naissance s'avère

\footnotetext{
${ }^{38}$ Voir Aurélie Houdebert, « Miniatures initiales et paroles inaugurales : naissance de l'œuvre, naissance du héros dans les manuscrits du Cléomadès ", infra, p. 109-124.

${ }^{39}$ Pierre Gibert, L'inconnue du commencement, Paris, Éditions du Seuil, coll. « La couleur des idées », 2007, p. 23 : «De soi, la naissance ne laisse aucun souvenir au nouveau-né. Pas seulement parce que le cerveau ne serait pas achevé à ce moment-là et ne disposerait pas des capacités de perception ou de mémorisation ultérieurement acquises, mais parce rien en moi, dans ma mémoire ou ma conscience, ne me permet d'en parler $»$.
} 
singulière $^{40}$. La contribution de Joanna Pavlevski dans ce bulletin en rend bien compte, examinant les relations entre texte et image dans les représentations de la naissance des enfants de Mélusine ${ }^{41}$.

Que ce soit dans la sphère sociale, comme dans la fiction, la naissance apparaît comme un événement à la fois préparé en amont - qu'il soit attendu ou au contraire différé - et entraînant en aval des transformations de l'espace, familial comme politique, qu'elle touche: moment a priori inaugural, la naissance nous semble bien souvent entraîner une dilation temporelle.

\section{Dilatations : temporalité complexe de la naissance}

Nous l'avons vu, la naissance ne saurait se résumer au moment de l'accouchement $^{42}$. Le processus pour qu'un individu vienne au monde est lent et progressif, il nécessite plusieurs étapes, chacune permettant la constitution de son identité. Cette construction débute en amont lors de la conception et de la grossesse et s'étend en aval aux cérémonies qui consacrent l'intégration du nouveau-né à la communauté. Cette extension de l'événement bien au-delà et en-deçà du jour où il se produit n'est pas

${ }^{40}$ Sur la question des naissances extraordinaires et monstrueuses, nous renvoyons à l'ouvrage de Maaike Van Der Lugt, Le ver, le démon et la Vierge. Les théories médiévales de la génération extraordinaire: une étude sur les rapports entre théologie, philosophie naturelle et médecine, Paris, Les Belles Lettres, coll. « L'âne d'or », 2004. Voir également Nicole Belmont, Les signes de la naissance. Études des représentations symboliques associées aux naissances singulières, Paris, Librairie Plon, 1971.

${ }^{41}$ Voir Joanna Pavlevski, « Naissances féeriques et fondation de lignée dans La Noble Histoire des Lusignan de Jean d'Arras et Le roman de Parthenay de Coudrette ", infra, p. 125-151.

${ }^{42}$ On notera qu'au Moyen Âge, on ne garde que rarement en mémoire sa date de naissance. Cette méconnaissance n'est pas tant due à une impossibilité de connaitre la date qu'à un manque d'intérêt pour cette information jusqu'à l'émergence de préoccupations astrologiques à partir du XIV ${ }^{\mathrm{e}}$ siècle. Emmanuel Poulle, « Temps des naissances ", dans Le temps, sa mesure et sa perception au Moyen Âge. Actes $d u$ colloque, Orléans, 12-13 avril 1991, dir. Bernard Ribémont, Caen, Paradigme, 1992, p. 205-215. 
sans rappeler la structure du cycle liturgique où Noël est précédé de l'Avent et dure jusqu'à l'Épiphanie tandis que Pâques est préparé par le Carême et ne s'achève que cinquante jours plus tard, lors de la Pentecôte.

Cet intervalle qui prépare la venue au monde de l'enfant est parsemé de signes qui annoncent sa destinée. C'est le temps des présages dont la littérature, à la suite de la Bible ou de la mythologie antique, est friande, tant dans les romans que dans l'hagiographie. Le destin de l'enfant à naître peut être connu par des visions ou des songes et le choix du prénom est souvent une façon de le vouer à sa future carrière.

Cette dilatation du temps de la génération se perçoit, par exemple, dans le récit de la naissance de saint Dominique telle que la rapporte Jacques de Voragine dans sa légende dorée :

Avant sa naissance, sa mère vit en songe qu'elle portait dans son sein un petit chien tenant dans sa gueule une torche allumée avec laquelle il embrasait tout l'univers. Quand elle l'eut mis au monde, une dame qui l'avait levé des fonts sacrés $\mathrm{du}$ baptême crut voir sur le front du petit Dominique une étoile très brillante qui éclairait toute la terre ${ }^{43}$.

Ici, rien n'est dit de l'accouchement lui-même. Les deux moments privilégiés sont ceux de la grossesse et du baptême. Le songe de la mère renvoie à l'étymologie, sous forme de jeu de mot, du prénom Dominique : domini canis, le chien de Dieu, et annonce la vocation à l'évangélisation du saint avec l'image de la torche ${ }^{44}$. Après l'accouchement, la naissance n'est achevée que lorsque l'enfant est baptisé. La vision lors du baptême de Dominique annonce à nouveau la vocation d'évangélisateur du saint. Les

43 Jacques de Voragine, La légende dorée, trad. Jean-Baptiste Marie Roze, Paris, Garnier-Flammarion, 1967, vol. 2, p. 46.

${ }^{44} \mathrm{Ce}$ genre de songe est courant dans l'hagiographie, on le retrouve dans les Vies de saint Bernard ou de saint François d'Assise par exemple. Voir André Vauchez, «Les saintes comme mères dans l'hagiographie occidentale $\left(\mathrm{X}^{\mathrm{e}}-\mathrm{XV}^{\mathrm{e}}\right.$ siècles $) »$, Micrologus: Natura, scienze e società medievali. Nature, Sciences and Medieval Societies, 2009, XVII : « La Madre / The Mother », p. 199-214, p. 203. 
deux médiatrices de ces visions sont la mère et la marraine; ainsi, le processus est commencé par la parenté biologique mais parachevé par la parenté spirituelle.

L'ensemble de l'épisode montre bien que la construction de l'identité et de la vocation de l'individu est un phénomène progressif dont le baptême est l'aboutissement. Nous retrouvons là cette cérémonie, fondamentale à plus d'un titre dans la société médiévale ${ }^{45}$. Elle apparaît comme une seconde naissance, naissance sociale qui dote l'enfant d'un réseau de parenté supplémentaire en la personne de ses parrains et marraines. Cette parenté de substitution est indispensable car, traditionnellement, les parents sont exclus de la cérémonie du baptême comme ceux qui ont transmis le péché originel à leur enfant ${ }^{46}$. Cela témoigne de la prééminence accordée à la parenté spirituelle sur la parenté charnelle. L'enfant reçoit également son nom au cours du baptême. Ce nom peut lui être donné par un des parrains et le lie à un saint patron et à une famille physique mais aussi spirituelle.

Si l'accouchement est le moment où l'enfant entre dans le monde, son entrée dans l'histoire ne commence qu'avec le baptême. En effet, l'incarnation du Christ change profondément la conception de l'histoire qui n'est plus cyclique, telle que la concevaient les philosophes de l'antiquité, mais qui est dès lors orientée vers une fin, annoncée dans l'Apocalypse. Chaque histoire individuelle devient une histoire sainte, temps de la conversion destiné à obtenir le Salut ${ }^{47}$. Le baptême est le point d'entrée dans cette histoire, première participation à la communauté chrétienne.

\footnotetext{
${ }^{45}$ Voir la première partie de notre introduction, p. 20.

${ }^{46}$ Jérôme Baschet, La civilisation féodale. De l'an mil à la colonisation de l'Amérique, Paris, Flammarion, coll. « Champs Histoire », p. 363.

${ }^{47}$ Jacques Fontaine, «La pédagogie augustinienne des rythmes du temps dans les Enarrationes in psalmos", dans Le temps chrétien. De la fin de l'Antiquité au Moyen Âge, III ${ }^{e}-X I I I^{e}$ siècle. Paris, 9-12 mars 1981, éd. Jean-Marie Leroux, Paris, Éditions du CNRS, coll. «Colloques internationaux du Centre National de la Recherche Scientifique », 604, 1984, p. 369-382.
} 
La cérémonie du baptême de l'enfant se double d'une cérémonie destinée à la mère : les relevailles dont la pratique se répand à partir du $\mathrm{XII}^{\mathrm{e}}$ siècle ${ }^{48}$. Quarante jours après l'accouchement, la jeune mère prenait le chemin de l'église sur le seuil de laquelle elle était accueillie par le prêtre qui lui permettait d'y entrer à nouveau. Si cette cérémonie renvoie aux règles touchant l'impureté féminine qui justifiaient qu'une femme ayant ses règles ne puisse approcher de l'autel, elle illustre aussi le fait que la naissance a mis la femme à l'écart de la société, l'a confiné dans la sphère privée et féminine. Une cérémonie est nécessaire à sa réintégration. La psychologie pourrait voir dans cette cérémonie une façon de manifester la nécessité de rompre le couple mère-enfant pour restituer sa place au père et permettre l'autonomisation progressive du nouveau-né.

Dans le domaine de la création littéraire, les «seuils de l'œuvre » sont souvent l'occasion pour l'auteur de mettre en scène le processus créatif, et la naissance peut alors servir de métaphore, plus ou moins explicite, pour décrire la production : conception, en amont, génération et gestation, et, en aval, présentation de l'œuvre au public, dans l'attente d'une reconnaissance esthétique ${ }^{49}$. Dans notre bulletin, précisément, Aurélie Houdebert examine, à partir des manuscrits du Cléomadès, l'articulation entre la naissance de l'œuvre, dans le prologue, et la naissance du héros, dans l'incipit narratif ${ }^{50}$. Quand s'opère l'émergence d'une conscience d'auteur, on retrouve cette dilatation du temps : comme l'a montré Barbara Falleiros pour Christine de Pizan, chaque œuvre devient

\footnotetext{
${ }^{48}$ Voir Becky R. Lee, «The purification of women after childbirth. A window onto medieval perceptions of women ", Florilegium. Carleton University Annual Papers on Classical Antiquity and the Middle Ages, 1996, vol. 14, p. 43-55.

${ }^{49}$ Nous renvoyons à Seuils de l'ouvre dans le texte médiéval, vol. I et II, études recueillies par Emmanuèle Baumgartner et Laurence Harf-Lancner, Paris, Presses de la Sorbonne Nouvelle, 2002.

${ }^{50}$ Aurélie Houdebert, art. cit.
} 
une occasion de se redéfinir, de proposer au lecteur une nouvelle naissance de l'auteur et de nouveaux contours à sa créativité ${ }^{51}$.

\section{Représenter et écrire la naissance : en quête de modèles}

La naissance représente ainsi un passage pour le couple qui acquiert le statut de parents et la respectabilité qui s'attache à ceux qui ont établi une famille et, là encore, la société intervient pour proposer des guides aux parents, pour normer leur comportement et les intégrer à la communauté ${ }^{52}$. Toutefois le christianisme, grand pourvoyeur de modèles et de normes semble étonnamment avare pour les parents, tant du côté de la mère que du père. Cela s'explique par la nature même du dieu chrétien : défini comme père, il revendique une filiation avec chaque homme, dévalorisant du même coup la parenté terrestre ${ }^{53}$. Si l'homme médiéval appelle les prêtres «mon père » et entend des prêches sur la mère Église, il ne peut pas en espérer un guide quant à ses pratiques quotidiennes, tant le vocabulaire de la parenté est alors utilisé pour désigner une réalité tout autre ${ }^{54}$.

Premier modèle pour les mères, la Vierge fournit une image riche d'ambiguïtés par le caractère extraordinaire de la conception de son enfant. Réalisant l'impossible, elle devient mère tout en conservant son précédent statut sexuel. Le culte qui rend hommage à sa maternité est une lente construction qui n'aboutit qu'à partir du XIII ${ }^{\mathrm{e}}$ siècle $^{55}$. À la même époque,

${ }^{51}$ Barbara Falleiros, art. cit.

${ }^{52}$ Les préceptes de la puériculture contemporaine ont été étudiés par une ethnologue et une psychanalyste qui exposent la façon dont la société impose sa norme aux comportements des parents, encore aujourd'hui. Voir Geneviève Delaisi de Parseval et Suzanne Lallemand, L'Art d'accommoder les bébés [1980], Paris, Éditions Odile Jacob, coll. «Poche Odile Jacob», 46, 2010. Nul doute que ces mécanismes existaient déjà au Moyen Âge.

${ }^{53}$ Jérôme Baschet, La civilisation féodale..., op. cit., p. 638.

${ }^{54}$ Ibid., p. 655-656.

${ }^{55}$ Claire Thiellet, «La dévotion mariale en Occident autour de l'an Mil», dans La Dévotion mariale de l'an mil à nos jours, dir. Bruno Béthouart et Alain Lottin, Arras, Artois Presses Université, coll. « Histoire », 2005, p. 75-80. 
Marie sert également de modèle à des femmes qui ont renié la maternité physique comme Héloïse ${ }^{56}$ ou à qui la nature a refusé la conception d'un enfant comme Jeanne de France $^{57}$, ce qui souligne assez qu'elle ne peut servir de point de repère aux simples mères. « Il y a [en effet] là affirmation d'un écart très fort et même d'un véritable renversement par rapport à l'ordre humain. Mais ce n'est pas le seul, car la naissance virginale se double de la réversibilité de la relation d'engendrement, et donc de la filiation : la Vierge est certes la mère du Christ, mais elle est également sa fille ; de manière symétrique et inverse, le Christ est à la fois le fils de la vierge et son père ${ }^{58} »$.

Cette difficulté à penser la maternité par l'Église se retrouve dans l'incompatibilité entre le statut de sainte et celui de mère. La sainteté féminine se définit avant tout par le statut sexuel de la femme, par sa virginité, alors que la sainteté masculine est définie par l'appartenance à un ordre (confesseur, évêque, prêtre, etc.). Outre les questions de pureté sexuelle, la maternité et les enfants solliciteraient trop la femme, la détourneraient de Dieu, l'empêchant de devenir la fiancée du Christ. Ainsi les mères qui deviennent saintes ne peuvent atteindre ce statut sans abandonner leur progéniture. C'est le cas de Juette d'Huy (1158-1228), d'Umiliana dei Cerchi (1219-1246) ou encore de Marguerite de Cortone ${ }^{59}$.

Mais les hommes sont encore moins bien lotis. Dans une religion où le Père par excellence est Dieu, il serait pour le moins présomptueux de se proposer un tel modèle. Reste donc le mari de la mère du Christ. Exemple impossible s'il en est dans une civilisation pour laquelle la légitimité de la

\footnotetext{
${ }^{56}$ Voir dans ce bulletin la contribution de Thibaut Randomme, art. cit.

${ }^{57}$ Voir dans ce bulletin la contribution de Stéphanie Richard, art. cit.

${ }^{58}$ Anita Guerreau-Jalabert, art. cit., p. 146.

${ }^{59}$ André Vauchez, «Les saintes comme mères dans l'hagiographie occidentale $\left(\mathrm{X}^{\mathrm{e}}-\right.$ $\mathrm{XV}^{\mathrm{e}}$ siècles) », art. cit., p. 205-206.
} 
filiation joue un rôle si important. Cela explique le caractère tardif du culte à Joseph qui n'apparait qu'au XIV ${ }^{\mathrm{e}}$ siècle et reste fort discret ${ }^{60}$.

C'est la figure du patriarche Abraham qui longtemps illustra le mieux la paternité. $\mathrm{Du} \mathrm{XI}^{\mathrm{e}}$ au $\mathrm{XIII}^{\mathrm{e}}$ siècle, on trouve nombre de représentations d'Abraham accueillant en son sein les âmes du paradis. Mais là encore cette image est loin d'être univoque puisque la posture d'Abraham copie celle de la Vierge, que le père se présente en un geste maternel. Ce n'est pas une paternité biologique qui est ainsi exaltée mais au contraire une paternité spirituelle ${ }^{61}$.

Le caractère équivoque des modèles que convoque le Moyen Âge pour représenter la naissance se retrouve dans le domaine de la création poétique. Le Roman de la Rose de Jean de Meun, notamment, illustre la nécessité du détour par l'image et le mythe pour dire et écrire la génération, de l'homme comme de l'œuvre: les récits de naissances mythologiques s'associent aux métaphores de l'écriture comme procréation, pour tenter de saisir par le discours l'acte poétique, au sens étymologique du terme. De fait, l'ambigüité intrinsèque de la naissance, évidente et universelle et dans le même temps insaisissable et polysémique, offre à l'écrivain une plasticité féconde. Comme le montre Didier Lechat, Génius « unit dans un même faisceau métaphorique l'usage du stylet sur les tablettes, celui du marteau sur l'enclume et celui des socs de la charrue dans la terre: équivoques filées entre les activités créatrices de l'homme et l'acte de procréation $^{62} »$. C'est également ce détour par le trope qui permet à

${ }^{60}$ Voir Paul Payan, Joseph. Une image de la paternité dans l'Occident médiéval, Paris, Aubier, coll. « Collection historique », 2006.

${ }^{61}$ Nous renvoyons à Jérôme Baschet, Le sein du père. Abraham et la paternité dans l'Occident médiéval, Paris, Gallimard, coll. « Le Temps des images », 2000.

${ }^{62}$ Didier Lechat, Dire par fiction : métamorphoses du je chez Guillaume de Machaut, Jean Froissart et Christine de Pizan, Paris, Champion, 2005, p. 385. Voir également l'article de Jacqueline Cerquiglini-Toulet, " L'inspiration des poètes lyriques à la fin du Moyen Âge: le cas de Christine de Pizan », dans L'inspiration. Le souffle créateur dans les arts, littératures et mystiques du Moyen Âge européen et proche- 
Christine de Pizan de reconstruire a posteriori sa naissance de femme écrivain et d'affirmer son accès à l'existence poétique. L'enjeu est de taille : les images de génération et d'engendrement, rapprochant la création artistique de la naissance, confèrent une épaisseur, et par là une légitimité, au travail de l'auteur.

Voici donc les jalons qui ont guidé notre réflexion sur la naissance, suscitée et nourrie par de récents et riches travaux sur les représentations de la parenté et de la filiation, auxquels les jeunes chercheurs de «Questes » espèrent, avec ce bulletin, apporter leur humble pierre ${ }^{63}$.

oriental. Colloque international tenu en Sorbonne les 23-24 mai 2002, éd. Claire Kappler et Roger Grozelier, Paris/Budapest/Kinshasa, L'Harmattan, coll. « Kubaba Actes », 8, 2006, p. 291-302.

${ }^{63}$ Aux ouvrages et travaux que l'on a cités dans cette introduction, l'on peut ajouter les deux journées d'étude internationales qui ont eu lieu tout récemment sur le thème « Naissance et petite enfance à la cour de France (Moyen Âge-XIX ${ }^{\mathrm{e}}$ siècle)», organisées par Stanis Pérez et Caroline Zum Kolk les 27 et 28 février 2014 à la Maison des Sciences de l'Homme Paris Nord, dans le cadre du projet scientifique Cour de France.fr et avec la collaboration du programme inter-universitaire «Formes du savoir de 1400 à $1750 »$ (Maison des Sciences de l'Homme Aquitaine). 